\title{
Unstable geodesics and topological field theory
}

\author{
Yukinori Yasui* \\ Depertment of Physics, Osaka City University, \\ Sumiyoshiku, Osaka, Japan \\ Satoshi Takahashi ${ }^{\dagger}$ \\ Depertment of Mathematics, Osaka City University, \\ Sumiyoshiku, Osaka, Japan
}

December 1993

\begin{abstract}
A topological field theory is used to study the cohomology of mapping space. The cohomology is identified with the BRST cohomology realizing the physical Hilbert space and the coboundary operator given by the calculations of tunneling between the perturbative vacua. Our method is illustrated by a simple example.
\end{abstract}

\footnotetext{
*E-mail address: f51999@sakura.kudpc.kyoto-u.ac.jp

${ }^{\dagger}$ E-mail address: e50874@JPNKUDPC.BITNET
} 


\section{INTRODUCTION}

Recently much attension has been paid on the study of topological quantum field theories. ${ }^{1,2}$ In this paper, we discuss a topological field theory over 2dimensional space $\Sigma=\mathbb{R} \times[0,1]$ and give a field theoretic realization of the cohomology of the mapping space $\Omega_{p, q}(N)=\{\phi:[0,1] \longrightarrow N \mid \phi(0)=$ $p, \phi(1)=q\}$ for a Riemannian manifold $(N, g)$, which arises as the BRST cohomology representing the physical Hilbert space.

The techniques we employ to evaluate the BRST cohomology are a mixture of Morse theory adapted to $\Omega_{p, q}(N)$ and instanton calculations - calculations of tunneling between the perturbative vacua of the topological theory. This approach was initiated by Witten in finite-dimensional situations. ${ }^{3}$ Using a supersymmetric quantum mechanics, he introduced a new cohomology complex and provided a path integral method of computing the cohomology of a finite-dimensional manifold $M$.

Let us summarize Witten's idea. Suppose we are given the following data concerning Morse theory:

(M-1) a Morse function $E$ on $M$,

(M-2) the critical points of $E$,

(M-3) the Morse index of each critical point, i.e., the number of negative eigenvalues of the Hessian of $E$ evaluated at the critical point.

Once we have these data, we can construct the perturbative vacuum for each critical point. The vacua are represented as forms on $M$, whose degrees are equal to the Morse indices of the corresponding critical points. Then we define, for each degree $\ell$, the vector space $X^{\ell}$ consisting of $\ell$-form vacua. Under fortunate circumstances (for example there are no vector spaces of odd indices), each vacuum becomes a generator of the cohomology of $M$. However, we must in general consider a coboundary operator $\delta$ : $X^{\ell} \longrightarrow X^{\ell+1}$ such that $\delta \circ \delta=0$ and

$$
H^{*}(M, \mathbb{R})=\operatorname{Ker} \delta / \operatorname{Im} \delta .
$$

This operator is interpreted as tunneling effects in a supersymmetric quantum mechanics and is evaluated by instanton calculations.

After Witten's work, some examples were explicitly calculated along this program and further the mathematical justification was established in terms of operator method. ${ }^{4-9}$ 
The main concern of this paper is therefore to describe a detailed construction of the coboundary operator when the manifold is taken to be the mapping space $\Omega_{p, q}(N)$, which is infinite-dimensional. In what follows, we are going to apply Witten's idea to $\Omega_{p, q}(N)$, but before we proceed, let us clarify the data concerning Morse theory. ${ }^{10}$

(M-1) One can define a functional on $\Omega_{p, q}(N)$ as follows,

$$
E[\phi]=\frac{1}{2} \int_{0}^{1} d x\left(\frac{\partial \phi}{\partial x}, \frac{\partial \phi}{\partial x}\right) \text { for } \phi \in \Omega_{p, q}(N) .
$$

This functional will play the role of the Morse function on $\Omega_{p, q}(N)$ when two points $p$ and $q$ on $N$ are not conjugate.

(M-2) The critical points of $E$ are the geodesics on $N$, where the gradient of $E, \operatorname{grad} E[\phi]=-\nabla_{x} \frac{\partial \phi}{\partial x}$, vanishes.

(M-3) The Hessian at the geodesic $\phi$ is the self-adjoint differential operator $J_{\phi}: \Gamma\left(\phi^{*} T N\right) \longrightarrow \Gamma\left(\phi^{*} T N\right)$ (Jacobi operator) defined by

$$
J_{\phi}=-\nabla_{x} \nabla_{x}-R\left(\bullet, \frac{\partial \phi}{\partial x}\right) \frac{\partial \phi}{\partial x} .
$$

Here we have used the following conventions; $(\bullet, \bullet)$ is the inner product with respect to the metric $g, \nabla_{x}$ the pull-back of the covariant derivative on $T N$ and $R$ the Riemannian curvature. ${ }^{11}$

This paper is organized as follows. In the next section we introduce the action of a topological field theory and discuss the instanton solutions, which are given by the solutions of a nonlinear heat equation. In section III, we describe the Hamiltonian formalism; the cohomology of the mapping space is identified with the BRST cohomology and the perturbative realization is investigated using normal coordinates on the mapping space. Section IV is concerned with the tunneling between the perturbative vacua, in which we calculate the matrix element of the BRST operator between the vacua, and determine the coboundary operator of a cohomology complex. In section $\mathrm{V}$ we study a simple example to illustlate our calculations. The final section is devoted to concluding remarks. Some of the detailed calculations are presented in the Appendix. 


\section{MODEL}

According to Langevin approach, ${ }^{12}$ we start with the action:

$$
\begin{aligned}
S_{0} & =\frac{1}{2} \int_{\Sigma} d t d x(K(\phi, G), K(\phi, G)), \\
K & =G-\frac{\partial \phi}{\partial t}+\operatorname{grad} E[\phi],
\end{aligned}
$$

where in local coordinates,

$$
\begin{gathered}
(K, K)=g_{i j}\left(G^{i}-\frac{\partial \phi^{i}}{\partial t}+\operatorname{grad} E[\phi]^{i}\right)\left(G^{j}-\frac{\partial \phi^{j}}{\partial t}+\operatorname{grad} E[\phi]^{j}\right), \\
\text { with } \operatorname{grad} E[\phi]^{i}=-\frac{\partial^{2} \phi^{i}}{\partial x^{2}}-\Gamma_{j k}^{i} \frac{\partial \phi^{j}}{\partial x} \frac{\partial \phi^{k}}{\partial x} .
\end{gathered}
$$

Here $\phi$ denotes the mapping $\Sigma \rightarrow N$ and $G \in \Gamma\left(\phi^{*} T N\right)$ the auxiliary field. The above action describes a system with no dynamical degrees of freedom: a shift of the field $G$ could eliminate all of the $\phi$ dependences. In such a situation, the Batalin-Vilkovisky algorithm enables us to construct a BRST invariant quantum action. ${ }^{13} \mathrm{~A}$ crucial feature of $S_{0}$ is that it has a local symmetry given by

$$
\begin{aligned}
\delta \phi^{i}= & \epsilon^{i} \\
\delta G^{i}= & \frac{\partial \epsilon^{i}}{\partial t}+\frac{\partial^{2} \epsilon^{i}}{\partial x^{2}}+2 \Gamma_{j k}^{i} \frac{\partial \phi^{k}}{\partial x} \frac{\partial \epsilon^{j}}{\partial x} \\
& +\partial_{j} \Gamma_{k \ell}^{i} \frac{\partial \phi^{k}}{\partial x} \frac{\partial \phi^{\ell}}{\partial x} \epsilon^{j}-\Gamma_{j k}^{i} K^{k} \epsilon^{j},
\end{aligned}
$$

where $\epsilon^{i}$ are local infinitesimal parameters of the transformation.

The algorithm proceeds with standard calculations; here we simply quote the result. The gauge-fixed Euclidian action is Q-exact:

$$
\begin{aligned}
S= & \int_{\Sigma} d t d x\left\{-\frac{1}{2}(B, B)-\left(\frac{\partial \phi}{\partial t}-\operatorname{grad} E[\phi], B\right)\right. \\
& \left.+\left(\psi, \nabla_{t} \psi^{*}-J_{\phi}\left(\psi^{*}\right)\right)+\frac{1}{4}\left(\psi, R\left(\psi^{*}, \psi^{*}\right) \psi\right)\right\} \\
= & \int_{\Sigma} d t d x Q\left(\psi, \frac{\partial \phi}{\partial t}-\operatorname{grad} E[\phi]+\frac{1}{2} B\right),
\end{aligned}
$$


with the BRST symmetry,

$$
\begin{aligned}
Q \phi^{i} & =-\psi^{* i} \\
Q \psi^{* i} & =0 \\
Q \psi_{i} & =-B_{i}+\psi_{j} \Gamma_{i k}^{j} \psi^{* k} \\
Q B_{i} & =-B_{j} \Gamma_{i k}^{j} \psi^{* k}+\frac{1}{2} \psi_{k} R^{k} i j \ell \psi^{* j} \psi^{* \ell} .
\end{aligned}
$$

Here $B$ and $\psi\left(\right.$ or $\left.\psi^{*}\right)$ are the auxiliary field and the ghost field in $\Gamma\left(\phi^{*} T N\right)$, respectively, and the covariant derivative,

$$
\nabla_{t} \psi^{* i}=\frac{\partial \psi^{* i}}{\partial t}+\Gamma_{j k}^{i} \frac{\partial \phi^{j}}{\partial t} \psi^{* k}
$$

is the pull-back of the covariant derivative on $T N$.

Furthermore, it is easy to see that the action is invariant (up to the surface term) under the discrete transformation,

$$
\psi \rightarrow \psi^{*}, \psi^{*} \rightarrow \psi, B \rightarrow B-2 \operatorname{grad} E[\phi]
$$

and

$$
E[\phi] \rightarrow-E[\phi] \quad\left(J_{\phi} \rightarrow-J_{\phi}\right)
$$

at the same time. As a result, the action Eq. (2.6) permits the second BRST symmetry $Q^{*}$, which is obtained by substituting Eqs. 2.9) and (2.10) into Eq.(2.7),

$$
\begin{aligned}
Q^{*} \phi^{i}= & -\psi^{i}, \\
Q^{*} \psi^{i}= & 0, \\
Q^{*} \psi_{i}^{*}= & -B_{i}+2 g_{i j} \operatorname{grad} E[\phi]^{j}+\psi_{j}^{*} \Gamma_{i k}^{j} \psi^{k}, \\
Q^{*} B_{i}= & 2 Q^{*}\left(g_{i j} \operatorname{grad} E[\phi]^{j}\right)-B_{j} \Gamma_{i k}^{j} \psi^{k} \\
& +2 g_{j k} \operatorname{grad} E[\phi]^{k} \Gamma_{i \ell}^{j} \psi^{\ell}+\frac{1}{2} \psi_{k}^{*} R_{i j \ell}^{k} \psi^{j} \psi^{\ell} .
\end{aligned}
$$

Using the equation of motion $\delta S / \delta B_{i}=0$, i.e.,

$$
B^{i}=-\frac{\partial \phi^{i}}{\partial t}+\operatorname{grad} E[\phi]^{i}
$$

we eliminate $B$ and the action takes the form,

$$
\begin{aligned}
S= & \int_{\Sigma} d t d x\left\{\frac{1}{2}\left(\frac{\partial \phi}{\partial t}, \frac{\partial \phi}{\partial t}\right)+\frac{1}{2}(\operatorname{grad} E[\phi], \operatorname{grad} E[\phi])\right. \\
& \left.+\left(\psi, \nabla_{t} \psi^{*}-J_{\phi}\left(\psi^{*}\right)\right)+\frac{1}{4}\left(\psi, R\left(\psi^{*}, \psi^{*}\right) \psi\right)\right\} .
\end{aligned}
$$


Before going into the quantization, we here consider the classical solutions (instantons) corresponding to tunneling processes. The geodesics on $N$, where $\operatorname{grad} E[\phi]=0$, can be regarded as the degenerate minima of the potential term,

$$
V[\phi]=\frac{1}{2} \int_{0}^{1} d x(\operatorname{grad} E[\phi], \operatorname{grad} E[\phi]) .
$$

Therefore, the relevant instantons are the steepest descent paths leading from one geodesic to another geodesic. They would be extrema of the bosonic part of the action,

$$
\begin{aligned}
S_{B}= & \int_{\Sigma} d t d x\left\{\frac{1}{2}\left(\frac{\partial \phi}{\partial t}, \frac{\partial \phi}{\partial t}\right)+\frac{1}{2}(\operatorname{grad} E[\phi], \operatorname{grad} E[\phi])\right\} \\
= & \int_{\Sigma} d t d x\left\{\frac{1}{2}\left(\frac{\partial \phi}{\partial t} \mp \operatorname{grad} E[\phi], \frac{\partial \phi}{\partial t} \mp \operatorname{grad} E[\phi]\right)\right. \\
& \left. \pm\left(\frac{\partial \phi}{\partial t}, \operatorname{grad} E[\phi]\right)\right\} .
\end{aligned}
$$

Let us fix the surface term in the above equation: $\phi(t, x) \in \Omega_{p, q}(N)$ for any fixed $t$. Then, the second term in $S_{B}$ is rewritten as follows:

$$
\begin{aligned}
\int_{\Sigma} d t d x\left(\frac{\partial \phi}{\partial t}, \operatorname{grad} E[\phi]\right) & =\int_{\Sigma} d t d x\left\{-\frac{d}{d x}\left(\frac{\partial \phi}{\partial t}, \frac{\partial \phi}{\partial x}\right)+\frac{1}{2} \frac{d}{d t}\left(\frac{\partial \phi}{\partial x}, \frac{\partial \phi}{\partial x}\right)\right\} \\
& =E[\phi(t=\infty)]-E[\phi(t=-\infty)],
\end{aligned}
$$

and hence we obtain an inequality,

$$
S_{B} \geq|E[\phi(t=\infty)]-E[\phi(t=-\infty)]| .
$$

The configurations that minimize the action are given by the solutions of a nonlinear heat equation,

$$
\frac{\partial \phi}{\partial t}= \pm \operatorname{grad} E[\phi]
$$

or in terms of the local coordinates,

$$
\frac{\partial \phi^{i}}{\partial t}=\mp\left(\frac{\partial^{2} \phi^{i}}{\partial x^{2}}+\Gamma_{j k}^{i} \frac{\partial \phi^{j}}{\partial x} \frac{\partial \phi^{k}}{\partial x}\right) .
$$

In particular, the solutions connecting geodesics express the steepest descent paths, and we call positive (negative) gradient flows instantons (antiinstantons). 
The next step is to investigate the space of these solutions. However, the direct study of the nonlinear heat equation is quite difficult and in general one can only prove that the instantons exist globally. ${ }^{14,15}$ Fortunately, for our purpose, the explicit form of the solutions is not necessary.

For each geodesic $\gamma$ with the Morse index $\ell_{\gamma}$ in $\Omega_{p, q}(N)$, we associate a variety $V_{\gamma}^{(+)}\left(V_{\gamma}^{(-)}\right)$, which is a union of the positive gradient solutions of Eq.2.18 departing from (arriving at) $\gamma ; V_{\gamma}^{(+)}\left(V_{\gamma}^{(-)}\right)$is a subvariety of codimension $\ell_{\gamma}$ (dimension $\ell_{\gamma}$ ) contained in $\Omega_{p, q}(N)$. To see local expression for the varieties, let us consider the tangent space at $\gamma$. Using the exponential mapping, $\exp _{\gamma(x)}: T_{\gamma(x)} N \longrightarrow N$, we write the general solution of Eq.(2.19) near $\gamma$ as follows,

$$
\phi(t, x)=\exp _{\gamma(x)} \xi(t, x), \quad \xi(t, x) \in T_{\gamma(x)} N .
$$

Substituting Eq.(2.20) into Eq.(2.19) and looking to the first order in $\xi$, we see that $\xi$ must satisfy

$$
\frac{\partial \xi}{\partial t}(t, x)=J_{\gamma} \xi(t, x),
$$

with the condition $\xi(t, 0)=\xi(t, 1)=0$ for all $t$. If we decompose $\xi$ into eigenfunctions of $J_{\gamma}$,

$$
\xi(t, x)=\sum_{\alpha=0}^{\infty} \xi^{\alpha}(t) e_{\alpha}(x), \quad J_{\gamma} e_{\alpha}(x)=\lambda_{\alpha} e_{\alpha}(x),
$$

Eq. 2.21) reduces to

$$
\frac{d \xi^{\alpha}}{d t}(t)=\lambda_{\alpha} \xi^{\alpha}(t) \quad \text { for all } \alpha
$$

These equations can be easily solved and determine the varieties $V_{\gamma}^{( \pm)}$near $\gamma$ :

$$
\begin{aligned}
& V_{\gamma}^{(+)}=\exp _{\gamma(x)}\left(\sum_{\lambda_{\alpha}>0} c_{\alpha}^{(+)} e^{\lambda_{\alpha} t} e_{\alpha}(x)\right) \text { for } t \rightarrow-\infty \\
& V_{\gamma}^{(-)}=\exp _{\gamma(x)}\left(\sum_{\lambda_{\alpha}<0} c_{\alpha}^{(-)} e^{\lambda_{\alpha} t} e_{\alpha}(x)\right) \text { for } t \rightarrow \infty
\end{aligned}
$$

with constants $c_{\alpha}^{( \pm)}$.

Let $\gamma_{A}$ and $\gamma_{B}$ be two geodesics with the Morse indices $\ell$ and $\ell+1$, respectively. Then, our assumptions are following: 
(A-1) There exist finite number of instantons connecting $\gamma_{A}$ with $\gamma_{B}$.

(A-2) $V_{\gamma_{A}}^{(+)}$and $V_{\gamma_{B}}^{(-)}$transversely intersect along these instantons.

Under these assumptions we write the moduli space of the instantons as,

$$
\mathcal{M}_{A B}=\bigcup_{\text {intersection }}\left(V_{\gamma_{A}}^{(+)} \cap V_{\gamma_{B}}^{(-)}\right)
$$

and deduce the dimension 1 .

(A-3) The tangent spaces along each instanton satisfy the transversality condition. This will be specified in section IV.

\section{HAMILTONIAN FORMALISM}

In this section we discuss the perturbative vacua for the system. This will be done in the Hamiltonian framework.

The canonical momenta and the Poisson bracket are readily read off from Eq.(2.13),

$$
p_{i}=\frac{\delta^{r} S}{\delta\left(\frac{\partial \phi^{i}}{\partial t}\right)}=g_{i j} \frac{\partial \phi^{j}}{\partial t}-\Gamma_{i j}^{k} \psi^{* j} \psi_{k}, \quad \psi_{i}=\frac{\delta^{r} S}{\delta\left(\frac{\partial \psi^{* i}}{\partial t}\right)}
$$

and

$$
\left\{\phi^{i}(x), p_{j}\left(x^{\prime}\right)\right\}=\delta_{j}^{i} \delta\left(x-x^{\prime}\right), \quad\left\{\psi^{* i}(x), \psi_{j}\left(x^{\prime}\right)\right\}=\delta_{j}^{i} \delta\left(x-x^{\prime}\right) .
$$

Then, the Hamiltonian corresponding to $S$ can be written as follows,

$$
\begin{aligned}
H= & \int_{0}^{1} d x\left\{\frac{1}{2}(\pi, \pi)-\frac{1}{2}(\operatorname{grad} E[\phi], \operatorname{grad} E[\phi])\right. \\
& \left.+\left(\psi, J_{\phi}\left(\psi^{*}\right)\right)-\frac{1}{4}\left(\psi, R\left(\psi^{*}, \psi^{*}\right) \psi\right)\right\},
\end{aligned}
$$

where $\pi_{i}=p_{i}+\Gamma_{i j}^{k} \psi^{* j} \psi_{k}$. The BRST charges are obtained by the Noether prescription and their action is given on using the Poisson bracket Eq.(3.2). In fact we find

$$
\begin{aligned}
Q & =\int_{0}^{1} d x\left(\psi^{*}, \pi-\operatorname{grad} E[\phi]\right), \\
Q^{*} & =\int_{0}^{1} d x(\psi, \pi+\operatorname{grad} E[\phi]),
\end{aligned}
$$


which satisfy the nilpotent condition,

$$
\{Q, Q\}=0, \quad\left\{Q^{*}, Q^{*}\right\}=0 .
$$

The Hamiltonian Eq. (3.3) may be rewritten as

$$
H=\frac{1}{2}\left\{Q, Q^{*}\right\},
$$

which is familiar with supersymmetric quantum theories.

Now let us proceed to the quantization. The classical phase space will be identified with the cotangent bundle of $\bigoplus_{r} \Lambda^{r}\left(T^{*} \Omega_{p, q}(N)\right)$. So the quantum Hilbert space $\mathcal{H}$ is the space of form-valued functions, i.e., $\mathcal{H}=\Gamma\left(\bigoplus_{r} \Lambda^{r}\left(T^{*} \Omega_{p, q}(N)\right)\right)$. Then the coordinate operators $\widehat{\phi}^{i}$ and $\widehat{\psi}^{* i}$ act as multiplication and exterior multiplication on the wave functions, respectively, while the momentum operators $\widehat{p}_{i}$ and $\widehat{\psi}_{i}$ act by differentiation, $\widehat{p}_{i} \sim \delta / \delta \phi^{i}$ and $\widehat{\psi}_{i} \sim \delta / \delta \psi^{* i}$ (interior product on forms).

We consider a local representation of the quantum Hilbert space. Let us first introduce a local coordinate system in $\Omega_{p, q}(N)$. For $\phi_{0} \in \Omega_{p, q}(N)$ an exponential mapping,

$$
\operatorname{Exp}_{\phi_{0}}: T_{\phi_{0}} \Omega_{p, q}(N)=\Gamma\left(\phi_{0}^{*} T N\right) \longrightarrow \Omega_{p, q}(N),
$$

is defined by $\operatorname{Exp}_{\phi_{0}}(X)=\exp \circ X$, where exp denotes the usual exponential mapping on $N$ and the symbol "O" the composition of mappings, i.e., for $X \in \Gamma\left(\phi_{0}^{*} T N\right), \quad X(x) \in T_{\phi_{0}(x)} N$ so that $\exp \circ X(x)=\exp _{\phi_{0}(x)} X(x) \in N$. Using an orthonormal basis $e_{\alpha}(\alpha=0,1, \ldots)$ of $\Gamma\left(\phi_{0}^{*} T N\right)$,

$$
\begin{aligned}
\left\langle e_{\alpha}, e_{\beta}\right\rangle & =\int_{0}^{1} d x\left(e_{\alpha}(x), e_{\beta}(x)\right) \\
& =\delta_{\alpha \beta},
\end{aligned}
$$

we have a normal coordinate system $\phi=\left(\xi^{0}, \xi^{1}, \ldots\right)$ with origin $\phi_{0}$ in $\Omega_{p, q}(N)$,

$$
\phi=\operatorname{Exp}_{\phi_{0}}(\xi)=\exp \circ \sum_{\alpha=0}^{\infty} \xi^{\alpha} e_{\alpha}, \quad \xi \in \Gamma\left(\phi_{0}^{*} T N\right) .
$$

Thus any wave function in $\mathcal{H}$ may be locally written in the form,

$$
\begin{aligned}
\omega & =\bigoplus_{r} \omega^{(r)}, \\
\omega^{(r)} & =\sum_{\alpha_{1}<\cdots<\alpha_{r}} \omega_{\alpha_{1} \cdots \alpha_{r}}(\xi) \psi^{* \alpha_{1}} \cdots \psi^{* \alpha_{r}} \in \Gamma\left(\Lambda^{r} T^{*} \Omega_{p, q}(N)\right),
\end{aligned}
$$


where we have identified $d \xi^{\alpha}$ with $\psi^{* \alpha}$.

The inner product on $\Gamma\left(\phi^{*} T N\right)$, in the normal coordinate system $\phi=$ $\operatorname{Exp}_{\phi_{0}}(\xi)$, is expressed as

$$
\begin{aligned}
G_{\alpha \beta} & =\left\langle\frac{\partial}{\partial \xi^{\alpha}}, \frac{\partial}{\partial \xi^{\beta}}\right\rangle \\
& =\int_{0}^{1} d x\left(\left.\frac{\partial}{\partial \lambda}\left(\exp \circ\left(\xi+\lambda e_{\alpha}\right)(x)\right)\right|_{\lambda=0},\left.\frac{\partial}{\partial \lambda}\left(\exp \circ\left(\xi+\lambda e_{\beta}\right)(x)\right)\right|_{\lambda=0}\right)
\end{aligned}
$$

and the expansion of $G_{\alpha \beta}$ takes the form (Appendix),

$$
G_{\alpha \beta}=\delta_{\alpha \beta}-\frac{1}{3} \int_{0}^{1} d x\left(R\left(e_{\alpha}, \xi\right) \xi, e_{\beta}\right)+\mathcal{O}\left(\xi^{3}\right) .
$$

Then $G^{\alpha \beta}=G_{\alpha \beta}^{-1}=\left\langle\psi^{* \alpha}, \psi^{* \beta}\right\rangle$, which may be extended to $\mathcal{H}$ : First define a product,

$$
\left\langle\psi^{* \alpha_{1}} \cdots \psi^{* \alpha_{p}}, \psi^{* \beta_{1}} \cdots \psi^{* \beta_{p}}\right\rangle=\sum_{r_{1}, \ldots, r_{p}} \epsilon_{r_{1} \cdots r_{p}} G^{\alpha_{1} \beta_{r_{1}}} \cdots G^{\alpha_{p} \beta_{r_{p}}},
$$

where $\left(r_{1}, \ldots, r_{p}\right)$ runs over $(1, \ldots, p)$ and

$$
\epsilon_{r_{1} \cdots r_{p}}=1(-1) \text { if }\left(\begin{array}{c}
1, \cdots, p \\
r_{1}, \cdots, r_{p}
\end{array}\right) \text { is even (odd). }
$$

It follows that

$$
\begin{gathered}
\left\langle\omega^{(r)}, \eta^{(r)}\right\rangle=\sum_{\alpha_{1}<\cdots<\alpha_{r}} \omega_{\alpha_{1} \cdots \alpha_{r}}(\xi) \eta^{\alpha_{1} \cdots \alpha_{r}}(\xi) \\
\text { for } \omega^{(r)}, \eta^{(r)} \in \Gamma\left(\Lambda^{r} T^{*} \Omega_{p, q}(N)\right)
\end{gathered}
$$

and a scalar product on $\mathcal{H}$ is formally given by

$$
\left\langle\omega^{(r)} \mid \eta^{(s)}\right\rangle=\delta_{r s} \int \prod_{\alpha} d \xi^{\alpha} \sqrt{G}\left\langle\omega^{(r)}, \eta^{(s)}\right\rangle .
$$

Now we discuss the BRST operators. These operators, which are quantum versions of Eqs. 3.4) and (3.5), will be written in the form,

$$
\begin{aligned}
\widehat{Q} & =\sum_{\alpha=0}^{\infty} \widehat{\psi}^{* \alpha}\left(\nabla \frac{\partial}{\partial \xi^{\alpha}}+\operatorname{grad} E[\phi]_{\alpha}\right), \\
\widehat{Q}^{*} & =\sum_{\alpha=0}^{\infty} G^{\alpha \beta}(\xi) \widehat{\psi}_{\beta}\left(-\nabla_{\frac{\partial}{\partial \xi^{\alpha}}}+\operatorname{grad} E[\phi]_{\alpha}\right) .
\end{aligned}
$$


Here $\nabla_{\frac{\partial}{\partial \xi^{\alpha}}}$ is the covariant derivative associated with the metric $G$ and

$$
\begin{aligned}
\operatorname{grad} E[\phi]_{\alpha} & =\left\langle\operatorname{grad} E[\phi], \frac{\partial}{\partial \xi^{\alpha}}\right\rangle \\
& =\int_{0}^{1} d x\left(-\nabla_{x} \frac{\partial \phi}{\partial x},\left.\frac{\partial}{\partial \lambda}\left(\exp \circ\left(\xi+\lambda e_{\alpha}\right)(x)\right)\right|_{\lambda=0}\right) .
\end{aligned}
$$

Then, we have the following expansion (Appendix):

$$
\operatorname{grad} E[\phi]_{\alpha}=\int_{0}^{1} d x\left\{\left(\operatorname{grad} E\left[\phi_{0}\right], e_{\alpha}\right)+\left(J_{\phi_{0}}(\xi), e_{\alpha}\right)\right\}+\mathcal{O}\left(\xi^{2}\right)
$$

By introducing exterior differential $d$ and formal adjoint $d^{*}$,

$$
\begin{aligned}
d & =\sum_{\alpha=0}^{\infty} \widehat{\psi}^{* \alpha} \nabla_{\frac{\partial}{\partial \xi^{\alpha}}}: \Gamma\left(\Lambda^{r} T^{*} \Omega_{p, q}(N)\right) \rightarrow \Gamma\left(\Lambda^{r+1} T^{*} \Omega_{p, q}(N)\right), \\
d^{*} & =-\sum_{\alpha=0}^{\infty} \widehat{\psi}^{\alpha} \nabla_{\frac{\partial}{\partial \xi^{\alpha}}}: \Gamma\left(\Lambda^{r+1} T^{*} \Omega_{p, q}(N)\right) \rightarrow \Gamma\left(\Lambda^{r} T^{*} \Omega_{p, q}(N)\right),
\end{aligned}
$$

the BRST operators is conveniently expressed as follows,

$$
\begin{aligned}
\widehat{Q} & =e^{-E[\phi]} d e^{E[\phi]}, \\
\widehat{Q}^{*} & =e^{E[\phi]} d^{*} e^{-E[\phi]},
\end{aligned}
$$

and hence the quantum Hamiltonian is given by

$$
2 \widehat{H}=\widehat{Q} \widehat{Q}^{*}+\widehat{Q}^{*} \widehat{Q} .
$$

These operators may be considered as an infinite-dimensional extension of Witten's cohomology operators in supersymmetric quantum mechanics on finite-dimensional manifolds. ${ }^{3}$

For the remainder of this section, we will discuss the physical Hilbert space $\mathcal{H}_{\text {phys }}$. This is usually defined by the BRST cohomology: the condition $\widehat{Q} \omega_{\text {phys }}=0, \omega_{\text {phys }} \in \mathcal{H}$ and furthermore $\omega_{\text {phys }}^{\prime}=\omega_{\text {phys }}+\widehat{Q}(\bullet)$ is equivalent to $\omega_{\text {phys }}$. By using the following complex,

$$
\begin{aligned}
& 0 \quad \longrightarrow \quad \Lambda^{0} T^{*} \Omega_{p, q}(N) \stackrel{d}{\longrightarrow} \Lambda^{1} T^{*} \Omega_{p, q}(N) \stackrel{d}{\longrightarrow} \quad \ldots \\
& \downarrow e^{-E[\phi]} \quad \downarrow e^{-E[\phi]}
\end{aligned}
$$




$$
0 \quad \longrightarrow \quad \Lambda^{0} T^{*} \Omega_{p, q}(N) \stackrel{\widehat{Q}}{\longrightarrow} \Lambda^{1} T^{*} \Omega_{p, q}(N) \stackrel{\widehat{Q}}{\longrightarrow} \cdots,
$$

$\mathcal{H}_{\text {phys }}$ is expressed as follows:

$$
\begin{aligned}
\mathcal{H}_{\text {phys }} & =\operatorname{Ker} \widehat{Q} / \operatorname{Im} \widehat{Q} \\
& \simeq \operatorname{Ker} \widehat{H} \\
& \simeq \operatorname{Ker} d / \operatorname{Im} d .
\end{aligned}
$$

These isomorphisms show that $\mathcal{H}_{\text {phys }}$ is the vector space of quantum vacua or equivalently the de Rham cohomology $H^{*}\left(\Omega_{p, q}(N)\right)$.

We now investigate the quantum vacua by a perturbative approximation. This is done by taking a normal coordinate system around each geodesic, which corresponds to the classical vacuum. Let $\gamma$ be a geodesic with the Morse index $\ell$ and $e_{\alpha} \in \Gamma\left(\gamma^{*} T N\right)(\alpha=0,1, \ldots)$ a basis consisting of the orthonormal eigenfunctions of $J_{\gamma}$ with the eigenvalues $\lambda_{\alpha}<0(\alpha=$ $0,1, \ldots, \ell-1)$ and $\lambda_{\alpha}>0(\alpha \geq \ell)$. Using the expansion formulas Eqs.(3.14) and (3.23), applied to the normal coordinate system $\phi=\operatorname{Exp}_{\gamma}\left(\sum_{\alpha=0}^{\infty} \xi^{\alpha} e_{\alpha}\right)$ where $\operatorname{grad} E[\gamma]=0$ and $J_{\gamma}\left(e_{\alpha}\right)=\lambda_{\alpha} e_{\alpha}$, we have approximate BRST operators in a neighborhood of $\gamma$,

$$
\begin{aligned}
& \widehat{Q}_{\gamma}=\sum_{\alpha=0}^{\infty} \widehat{\psi}^{* \alpha}\left(\frac{\partial}{\partial \xi^{\alpha}}+\lambda_{\alpha} \xi^{\alpha}\right), \\
& \widehat{Q}_{\gamma}^{*}=\sum_{\alpha=0}^{\infty} \widehat{\psi}_{\alpha}\left(-\frac{\partial}{\partial \xi^{\alpha}}+\lambda_{\alpha} \xi^{\alpha}\right),
\end{aligned}
$$

It readily follows that the corresponding Hamiltonian is

$$
\widehat{H}_{\gamma}=\sum_{\alpha=0}^{\infty}\left(-\left(\frac{\partial}{\partial \xi^{\alpha}}\right)^{2}+\left(\lambda_{\alpha} \xi^{\alpha}\right)^{2}+\lambda_{\alpha}\left[\widehat{\psi}^{* \alpha}, \widehat{\psi}_{\alpha}\right]\right)
$$

and the normalized vacuum state of $\widehat{H}_{\gamma}$, zero-eigenvalue function, is given by

$$
\Omega_{\gamma}=\prod_{\alpha}\left(\frac{\left|\lambda_{\alpha}\right|}{\pi}\right)^{\frac{1}{4}} e^{-\frac{1}{2}\left|\lambda_{\alpha}\right|\left(\xi^{\alpha}\right)^{2}} \omega^{(\ell)}[\varepsilon],
$$

where $\omega^{(\ell)}[\varepsilon]$ is the $\ell$-form with negative-eigenvalue indices in the frame $\varepsilon=\left(e_{0}, e_{1}, \ldots\right)$,

$$
\omega^{(\ell)}[\varepsilon]=\psi^{* 0} \psi^{* 1} \cdots \psi^{* \ell-1} .
$$


Let $C^{\ell}=\left\{\gamma_{I} \mid I=1,2, \ldots, n(\ell)\right\}$ be a set of geodesics with the Morse index $\ell$. For each $\gamma_{I} \in C^{\ell}$, we can assign a $\ell$-form vacuum $\Omega_{\gamma_{I}}$, which induces an orientation $\omega^{(\ell)}\left[\varepsilon^{I}\right]$ on the $\ell$-dimensional vector space $N\left(\gamma_{I}\right) \subset$ $\Gamma\left(\gamma_{I}^{*} T N\right)$ spanned by the negative-eigenvalue functions of $J_{\gamma_{I}}$. Then, $\mathcal{H}_{\text {phys }}$ is approximated by

$$
\mathcal{H}_{\text {phys }} \simeq \bigoplus_{\ell=0}^{\infty} X^{\ell},
$$

where $X^{\ell}$ is the $n(\ell)$-dimensional vector space spanned by $\Omega_{\gamma_{I}}$.

\section{TUNNELING CALCULATIONS}

In this section, we evaluate transition matrix elements between the perturbative vacua. These can be used to construct the coboundary operator $\delta^{(\ell)}: X^{\ell} \longrightarrow X^{\ell+1}$.

Suppose we have an instanton $\phi_{0}$ connecting two geodesics $\gamma_{A}$ and $\gamma_{B}$ with the Morse indices $\ell$ and $\ell+1$, respectively. As we saw in section II, the instanton has exactly one free parameter corresponding to the time translation. Then the vector field $\frac{\partial \phi_{0}}{\partial t}$ is in the kernel of the operator,

$$
\nabla_{t}-J_{\phi_{0}}: \Gamma\left(\phi_{0}^{*} T N\right) \longrightarrow \Gamma\left(\phi_{0}^{*} T N\right)
$$

and

$$
\int_{\Sigma} d t d x\left(\frac{\partial \phi_{0}}{\partial t}, \frac{\partial \phi_{0}}{\partial t}\right)=E\left[\gamma_{B}\right]-E\left[\gamma_{A}\right]<\infty .
$$

This means that there exists one ghost zero mode, which forces the partition function to vanish. An analogous situation appeared in supersymmetric quantum mechanics. ${ }^{3-8}$ So if we wish to calculate the nontrivial transition between the perturbative vacua, we must take the matrix element $\left\langle\Omega_{\gamma_{B}}|\widehat{Q}| \Omega_{\gamma_{A}}\right\rangle$, in which $\widehat{Q}$ absorbs the zero mode in the ghost path integral measure.

Using the commutation relation $[\widehat{Q}, f[\phi]]=d f[\phi]$ for any functional $f[\phi]$, and the fact $\left\langle\Omega_{\gamma_{I}}|d f[\phi]| \Omega_{\gamma_{I}}\right\rangle \simeq f\left[\gamma_{I}\right] \quad(I=A, B)$, we get

$$
\left\langle\Omega_{\gamma_{B}}|\widehat{Q}| \Omega_{\gamma_{A}}\right\rangle \simeq \frac{1}{f\left[\gamma_{A}\right]-f\left[\gamma_{B}\right]}\left\langle\Omega_{\gamma_{B}}|d f[\phi]| \Omega_{\gamma_{A}}\right\rangle .
$$

We now use standard path integral techniques. Let us consider the heat kernel with the insertion $d f$,

$$
K_{d f}\left[\gamma_{A} \psi_{A}, \gamma_{B} \psi_{B}^{*}\right] \in \operatorname{Hom}\left(\Lambda^{\ell} T_{\gamma_{A}}^{*} \Omega_{p, q}(N), \Lambda^{\ell+1} T_{\gamma_{B}}^{*} \Omega_{p, q}(N)\right),
$$


defined by the path integral

$$
K_{d f}\left[\gamma_{A} \psi_{A}, \gamma_{B} \psi_{B}^{*}\right]=\int[d \phi]\left[d \psi^{*}\right][d \psi] d f[\phi] e^{-S},
$$

where the boundary conditions are

$$
\begin{aligned}
& \phi(-\infty, x)=\gamma_{A}(x), \quad \phi(\infty, x)=\gamma_{B}(x), \\
& \psi(-\infty, x)=\psi_{A}(x) \quad \text { and } \quad \psi^{*}(\infty, x)=\psi_{B}^{*}(x) .
\end{aligned}
$$

The quantum effect of interest is the quadratic fluctuation around each instanton. We denote by $\phi_{s}$ a small deformation from an instanton $\phi$,

$$
\phi_{s}(t, x)=\exp _{\phi(t, x)} s \xi(t, x),
$$

where $s$ is the deformation parameter and

$$
\phi_{0}(t, x)=\phi(t, x),\left.\quad \frac{\partial \phi_{s}}{\partial s}(t, x)\right|_{s=0}=\xi(t, x) \in T_{\phi(t, x)} N .
$$

Using the expansion,

$$
\begin{aligned}
& \left(\frac{\partial \phi_{s}}{\partial t}-\operatorname{grad} E\left[\phi_{s}\right], \frac{\partial \phi_{s}}{\partial t}-\operatorname{grad} E\left[\phi_{s}\right]\right) \\
= & s^{2}\left(\nabla_{t} \xi-J_{\phi}(\xi), \nabla_{t} \xi-J_{\phi}(\xi)\right)+\mathcal{O}\left(s^{3}\right),
\end{aligned}
$$

we obtain the action up to the quadratic order :

$$
\begin{aligned}
S= & E\left[\gamma_{B}\right]-E\left[\gamma_{A}\right]+S^{(2)}+\text { higher order, } \\
S^{(2)}= & \int_{-T}^{T} d t \int_{0}^{1} d x\left\{\frac{1}{2}\left(\nabla_{t} \xi-J_{\phi}(\xi), \nabla_{t} \xi-J_{\phi}(\xi)\right)\right. \\
& +\left(\psi, \nabla_{t} \psi^{*}-J_{\phi}\left(\psi^{*}\right)\right\} \quad(T \rightarrow \infty) .
\end{aligned}
$$

The treatment of the path integral may be further simplified if the adiabatic approximation applies to Eq.(4.12). For this we change the integral variable $t=T \tau$ and $\xi(t, x)=\sqrt{T} \xi(\tau, x)$ at the same time, so that $S^{(2)}$ becomes

$$
\begin{aligned}
S^{(2)}= & \int_{-1}^{1} d \tau \int_{0}^{1} d x\left\{\frac{1}{2}\left(\nabla_{\tau} \xi-T J_{\phi}(\xi), \nabla_{\tau} \xi-T J_{\phi}(\xi)\right)\right. \\
& +\left(\psi, \nabla_{\tau} \psi^{*}-T J_{\phi}\left(\psi^{*}\right)\right\}
\end{aligned}
$$


Let us expand the tangent vectors $\xi, \psi$ and $\psi^{*}$ in terms of the eigenfunctions of $J_{\phi}$,

$$
\begin{gathered}
\xi(\tau, x)=\sum_{\alpha=0}^{\infty} \xi^{\alpha}(\tau) u_{\alpha}^{T}(\tau, x), \\
\psi(\tau, x)=\sum_{\alpha=0}^{\infty} \psi^{\alpha}(\tau) u_{\alpha}^{T}(\tau, x), \\
\psi^{*}(\tau, x)=\sum_{\alpha=0}^{\infty} \psi^{* \alpha}(\tau) u_{\alpha}^{T}(\tau, x),
\end{gathered}
$$

where

$$
J_{\phi} u_{\alpha}^{T}(\tau, x)=\lambda_{\alpha}^{T}(\tau) u_{\alpha}^{T}, \quad u_{\alpha}^{T}(\tau, x) \in T_{\phi(T \tau, x)} N
$$

with the normalization

$$
\int_{0}^{1} d x\left(u_{\alpha}^{T}, u_{\beta}^{T}\right)=\delta_{\alpha \beta}
$$

A perturbative analysis yields that the eigenfunctions $u_{\alpha}^{T}$ and the corresponding eigenvalues $\lambda_{\alpha}^{T}$ have the behavior in the large limit $T$,

$$
\begin{aligned}
u_{\alpha}^{T}(\tau, x) & \sim\left\{\begin{array}{l}
u_{\alpha}^{A}(x)+\sum_{\beta \neq \alpha}\left(\frac{1}{\lambda_{\beta}^{A}-\lambda_{\alpha}^{A}} \sum_{\lambda_{\gamma}^{A}>0} c_{\alpha \beta \gamma}^{A} e^{\lambda_{\gamma}^{A} T \tau}\right) u_{\beta}^{A}(x) \quad \text { for } \quad \tau<0, \\
u_{\alpha}^{B}(x)+\sum_{\beta \neq \alpha}\left(\frac{1}{\lambda_{\beta}^{B}-\lambda_{\alpha}^{B}} \sum_{\lambda_{\gamma}^{B}<0} c_{\alpha \beta \gamma}^{B} e^{\lambda_{\gamma}^{B} T \tau}\right) u_{\beta}^{B}(x) \quad \text { for } \quad \tau>0
\end{array}\right. \\
\lambda_{\alpha}^{T}(\tau) & \sim \begin{cases}\lambda_{\alpha}^{A}+\sum_{\lambda_{\gamma}^{A}>0} c_{\alpha \alpha \gamma}^{A} e^{\lambda_{\gamma}^{A} T \tau} & \text { for } \quad \tau<0 \\
\lambda_{\alpha}^{B}+\sum_{\lambda_{\gamma}^{B}<0} c_{\alpha \alpha \gamma}^{B} e^{\lambda_{\gamma}^{B} T \tau} & \text { for } \quad \tau>0\end{cases}
\end{aligned}
$$

where $u_{\alpha}^{I}(\alpha=0,1, \ldots ; I=A, B)$ are the eigenfunctions of $J_{\gamma_{I}}$ with the eigenvalues $\lambda_{\alpha}^{I}$ and

$$
\begin{aligned}
c_{\alpha \beta \gamma}^{I}= & \int_{0}^{1} d x\left\{\left(\left(\nabla_{u_{\gamma}^{I}} R\right)\left(u_{\alpha}^{I}, \frac{d \gamma_{I}}{d x}\right) \frac{d \gamma_{I}}{d x}, u_{\beta}^{I}\right)+\left(\left(\nabla_{x} R\right)\left(u_{\gamma}^{I}, \frac{d \gamma_{I}}{d x}\right) u_{\alpha}^{I}, u_{\beta}^{I}\right)\right. \\
& \left.+2\left(R\left(u_{\alpha}^{I}, \frac{d \gamma_{I}}{d x}\right) \nabla_{x} u_{\gamma}^{I}, u_{\beta}^{I}\right)+2\left(R\left(u_{\gamma}^{I}, \frac{d \gamma_{I}}{d x}\right) \nabla_{x} u_{\alpha}^{I}, u_{\beta}^{I}\right)\right\}, \quad \text { (4.21) }
\end{aligned}
$$

which are T-independent constants. 
By substituting Eqs.(4.14), (4.15) and (4.16) into Eq.4.13), the quadratic action takes the form

$$
\begin{aligned}
S^{(2)}= & \int_{-1}^{1} d \tau\left\{\frac{1}{2} \sum_{\alpha}\left(\frac{d \xi^{\alpha}}{d \tau}+\sum_{\beta} \xi^{\beta} \omega_{\beta \alpha}^{T}-T \lambda_{\alpha}^{T} \xi^{\alpha}\right)^{2}\right. \\
& \left.+\sum_{\alpha} \psi^{\alpha}\left(\frac{d \psi^{* \alpha}}{d \tau}+\sum_{\beta} \psi^{* \beta} \omega_{\beta \alpha}^{T}-T \lambda_{\alpha}^{T} \psi^{* \alpha}\right)\right\} .
\end{aligned}
$$

Here the connection $\omega_{\alpha \beta}^{T}$ is defined by

$$
\omega_{\alpha \beta}^{T}(\tau)=\int_{0}^{1} d x\left(\nabla_{\tau} u_{\alpha}^{T}(\tau, x), u_{\beta}^{T}(\tau, x)\right)=-\omega_{\beta \alpha}^{T}(\tau),
$$

which behaves for large $T$ like

$$
\omega_{\alpha \beta}^{T}(\tau) \sim \begin{cases}\frac{1}{\lambda_{\beta}^{A}-\lambda_{\alpha}^{A}} \sum_{\lambda_{\gamma}^{A}>0} c_{\alpha \beta \gamma}^{A} \lambda_{\gamma}^{A} T e^{\lambda_{\gamma}^{A} T \tau} & \text { for } \quad \tau<0, \\ \frac{1}{\lambda_{\beta}^{B}-\lambda_{\alpha}^{B}} \sum_{\lambda_{\gamma}^{B}<0} c_{\alpha \beta \gamma}^{B} \lambda_{\gamma}^{B} T e^{\lambda_{\gamma}^{B} T \tau} & \text { for } \quad \tau>0 .\end{cases}
$$

So comparing this formula Eq.4.24 with Eq.4.20 leads to an inequality,

$$
\left|\omega_{\alpha \beta}^{T}(\tau)\right| \ll\left|T \lambda_{\alpha}^{T}(\tau)\right| \quad \text { for large } T \quad(\tau \neq 0) .
$$

This implies that the connection terms in Eq. (4.22) can be neglected for the large limit $T$. In other words, the eigenfunctions $u_{\alpha}^{T}$ are covariant constants with respect to $\tau$ within this approximation.

Returning to the original variable $t$, i.e., $\xi^{\alpha}(t)=\sqrt{T} \xi^{\alpha}(\tau), \lambda_{\alpha}(t)=\lambda_{\alpha}^{T}(\tau)$ and $u_{\alpha}(t, x)=u_{\alpha}^{T}(\tau, x)$, we get the approximate action,

$$
S^{(2)} \simeq \int_{-T}^{T} d t \sum_{\alpha}\left\{\frac{1}{2}\left(\frac{d \xi^{\alpha}}{d t}-\lambda_{\alpha} \xi^{\alpha}\right)^{2}+\psi^{\alpha}\left(\frac{d \psi^{* \alpha}}{d t}-\lambda_{\alpha} \psi^{* \alpha}\right)\right\}
$$

and the path integral becomes

$$
\begin{aligned}
& K_{d f}\left[\gamma_{A} \psi_{A}, \gamma_{B} \psi_{B}^{*}\right]=e^{-\left(E\left[\gamma_{B}\right]-E\left[\gamma_{A}\right]\right)} \times \\
& \sum_{\text {instanton }} \int[d \xi]\left[d \psi^{*}\right][d \psi]\left(\sum_{\alpha} \frac{\partial f}{\partial \xi^{\alpha}}[\phi] \psi^{* \alpha}\right) e^{-S^{(2)}} .
\end{aligned}
$$


Before going into the concrete calculation of Eq. (4.27), we make some comments concerning the assumption (A-3) in section II. Let us define the subsets of the indices $\mathcal{I}=\{\alpha=0,1, \ldots\}$ in the eigenvalues $\lambda_{\alpha}(t)$,

$$
\mathcal{J}^{(+)}=\left\{\alpha \mid \lambda_{\alpha}(-t)>0\right\}, \quad \mathcal{J}^{(-)}=\left\{\alpha \mid \lambda_{\alpha}(t)<0\right\} \quad \text { for } \quad t \rightarrow \infty .
$$

Then, (A-3) means that the tangent spaces spanned by $u_{\alpha}(t, \bullet)\left(\alpha \in \mathcal{J}^{(+)}\right)$ and $u_{\alpha}(t, \bullet)\left(\alpha \in \mathcal{J}^{(-)}\right)$satisfy the transversality condition:

$$
\mathcal{I}=\mathcal{J}^{(+)} \cup \mathcal{J}^{(-)}
$$

and hence

$$
\sharp\left(\mathcal{J}^{(+)} \cap \mathcal{J}^{(-)}\right)=1, \quad \sharp\left(\mathcal{I} \backslash \mathcal{J}^{(+)}\right)=\ell .
$$

From now on we will write the indices as

$$
\begin{aligned}
\mathcal{I}^{(+)} & =\mathcal{I} \backslash \mathcal{J}^{(-)}=\{\ell+1, \ell+2, \ldots\}, \\
\mathcal{I}^{(-)} & =\mathcal{I} \backslash \mathcal{J}^{(+)}=\{0,1, \ldots, \ell-1\}, \\
\mathcal{I}^{(+-)} & =\mathcal{J}^{(+)} \cap \mathcal{J}^{(-)}=\{\ell\} .
\end{aligned}
$$

Note that $\mathcal{I}^{(+-)}$is the set associated with the zero mode.

Now let us turn to the explicit calculation of the path integral Eq.(4.27). We start with the evaluation of the bosonic fluctuations, which is the functional determinant of the differential operator such as

$$
\widehat{\mathcal{O}}_{\alpha}=-\left(\frac{d}{d t}+\lambda_{\alpha}(t)\right)\left(\frac{d}{d t}-\lambda_{\alpha}(t)\right) \quad \text { for } \quad \alpha \in \mathcal{I} .
$$

The determinant is easily calculated by the Gelfand-Yaglom method. ${ }^{16,17}$ Using the asymptotic behavior Eq.4.20), we get the formula up to the $\alpha$ independent normalization :

$$
\left(\operatorname{det} \widehat{\mathcal{O}}_{\alpha}\right)^{-\frac{1}{2}}=\left\{\begin{array}{lr}
\sqrt{\lambda_{\alpha}^{A}} c_{\alpha} e^{-\frac{T}{2}\left(\lambda_{\alpha}^{A}+\lambda_{\alpha}^{B}\right)} & \text { for } \quad \alpha \in \mathcal{I}^{(+)}, \\
\frac{\sqrt{\left|\lambda_{\alpha}^{B}\right|} c_{\alpha} e^{\frac{T}{2}\left(\lambda_{\alpha}^{A}+\lambda_{\alpha}^{B}\right)}}{1} & \text { for } \quad \alpha \in \mathcal{I}^{(-)} \\
\frac{1}{\sqrt{E\left[\gamma_{B}\right]-E\left[\gamma_{A}\right]}} \sqrt{\lambda_{\alpha}^{A}\left|\lambda_{\alpha}^{B}\right|} c_{\alpha} e^{-\frac{T}{2}\left(\lambda_{\alpha}^{A}-\lambda_{\alpha}^{B}\right)} & \text { for } \quad \alpha \in \mathcal{I}^{(+-)},
\end{array}\right.
$$

where $c_{\alpha}(\alpha \in \mathcal{I})$ are some constants, which can be eliminated by the contribution of the ghost fluctuations, and we have also removed the zero mode from the determinant in the third formula on the r.h.s.. 
Secondly, let us consider the contribution from the ghost fluctuations. This may be written as

$$
\int \prod_{\alpha \in \mathcal{I}}\left[d \psi^{* \alpha}\right]\left[d \psi^{\alpha}\right] \psi^{* \ell}(t) e^{-\int_{-T}^{T} d t \sum_{\alpha} \psi^{\alpha}\left(\frac{d}{d t} \psi^{* \alpha}-\lambda_{\alpha} \psi^{* \alpha}\right)}
$$

with B.C. Eq.(4.7).

It should be noticed that if there is no ghst insertion of $\mathcal{I}^{(+-)}$-component ( $\ell$ index), the path integral vanishes because of the zero mode as stated in the beginning of this section. The calculation of Eq. (4.36) is straightforward and we obtain

$$
\begin{aligned}
& \left(\prod_{\alpha \in \mathcal{I}} c_{\alpha}^{-1}\right) \int_{0}^{1} d x\left(u_{\ell}(t, x), \frac{\partial \phi}{\partial t}(t, x)\right)\left(\prod_{\alpha \in \mathcal{I}^{(+)}} e^{\frac{T}{2}\left(\lambda_{\alpha}^{A}+\lambda_{\alpha}^{B}\right)}\right) \\
& \times e^{\frac{T}{2}\left(\lambda_{\ell}^{A}-\lambda_{\ell}^{B}\right)}\left(\prod_{\alpha \in \mathcal{I}^{(-)}} e^{-\frac{T}{2}\left(\lambda_{\alpha}^{A}+\lambda_{\alpha}^{B}\right)}\right)\left(\psi_{B}^{* 0} \cdots \psi_{B}^{* \ell}\right)\left(\psi_{A}^{\ell-1} \cdots \psi_{A}^{0}\right) .
\end{aligned}
$$

The boundary term $\left(\psi_{B}^{* 0} \cdots \psi_{B}^{* \ell}\right)\left(\psi_{A}^{\ell-1} \cdots \psi_{A}^{0}\right)$ has the negative-eigenvalue indices, $\mathcal{I}^{(-)}$for $\psi_{A}$ and $\mathcal{I}^{(-)} \cup \mathcal{I}^{(+-)}$for $\psi_{B}^{*}$, and so it may be identified with (see Eq.(3.35))

$$
\omega^{(\ell+1)}\left[u_{\phi}^{B}\right] \otimes \omega^{(\ell)}\left[u_{\phi}^{A}\right]^{*} \in \Lambda^{\ell+1} T_{\gamma_{B}}^{*} \Omega_{p, q}(N) \otimes \Lambda^{\ell} T_{\gamma_{A}} \Omega_{p, q}(N),
$$

where $u_{\phi}^{A}$ and $u_{\phi}^{B}$ denote the frames of $\Gamma\left(\gamma_{A}^{*} T N\right)$ and $\Gamma\left(\gamma_{B}^{*} T N\right)$ defined by the eigenfunctions of $J_{\phi}, u_{\alpha}(t, x) \in T_{\phi(t, x)} N$ :

$$
\begin{aligned}
& u_{\phi}^{A}=\lim _{t \rightarrow-\infty}\left(u_{0}(t, x), u_{1}(t, x), \ldots\right)=\left(u_{0}^{A}(x), u_{1}^{A}(x), \ldots\right), \\
& u_{\phi}^{B}=\lim _{t \rightarrow \infty}\left(u_{0}(t, x), u_{1}(t, x), \ldots\right)=\left(u_{0}^{B}(x), u_{1}^{B}(x), \ldots\right) .
\end{aligned}
$$

These frames may be approximated by parallel transporting the frame $\left(u_{0}, u_{1}, \ldots\right)$ along the instanton $\phi$ since each vector $u_{\alpha}$ is almost covariant constant.

Putting everything together, we have the heat kernel,

$$
\begin{aligned}
K_{d f}\left[\gamma_{A} \psi_{A}, \gamma_{B} \psi_{B}^{*}\right]= & \frac{N}{\sqrt{2 \pi}} e^{-\left(E\left[\gamma_{B}\right]-E\left[\gamma_{A}\right]\right.}\left(f\left[\gamma_{B}\right]-f\left[\gamma_{A}\right]\right)\left(\prod_{\alpha \in \mathcal{I}^{(+)} \cup \mathcal{I}^{(+-)}} \sqrt{\lambda_{\alpha}^{A}}\right) \\
& \left(\prod_{\alpha \in \mathcal{I}^{(-)} \cup \mathcal{I}^{(+-)}} \sqrt{\left|\lambda_{\alpha}^{B}\right|}\right) \sum_{\phi} \omega^{(\ell+1)}\left[u_{\phi}^{B}\right] \otimes \omega^{(\ell)}\left[u_{\phi}^{A}\right]^{*}, \quad(4.41)
\end{aligned}
$$


where $N$ is the normalization constant and $\phi$ runs over the set of instantons connecting the two geodesics $\gamma_{A}$ and $\gamma_{B}$. Recall that

$$
\Omega_{\gamma_{I}}=\prod_{\alpha}\left(\frac{\left|\lambda_{\alpha}^{I}\right|}{\pi}\right)^{\frac{1}{4}} e^{-\frac{1}{2}\left|\lambda_{\alpha}^{I}\right|\left(\xi^{\alpha}\right)^{2}} \omega^{(m(I))}\left[\varepsilon^{I}\right]
$$

for the fixed frames of $\Gamma\left(\gamma_{I}^{*} T N\right), \quad \varepsilon^{I}=\left(e_{0}^{I}, e_{1}^{I}, \ldots\right) \quad(I=A, B ; m(A)=$ $\ell, m(B)=\ell+1$ ). From Eq.(4.3), after integrating $\Omega_{\gamma_{I}}$, we finally obtain the matrix element, ${ }^{18}$

$$
\begin{aligned}
\left\langle\Omega_{\gamma_{B}}|\widehat{Q}| \Omega_{\gamma_{A}}\right\rangle= & -\frac{1}{\pi} e^{-\left(E\left[\gamma_{B}\right]-E\left[\gamma_{A}\right]\right)}\left(\prod_{\alpha \in \mathcal{I}^{(+)} \cup \mathcal{I}^{(+-)}} \lambda_{\alpha}^{A}\right)^{\frac{1}{4}}\left(\prod_{\alpha \in \mathcal{I}^{(-)}}\left|\lambda_{\alpha}^{A}\right|\right)^{-\frac{1}{4}} \\
& \times\left(\prod_{\alpha \in \mathcal{I}^{(-)} \cup \mathcal{I}^{(+-)}}\left|\lambda_{\alpha}^{B}\right|\right)^{\frac{1}{4}}\left(\prod_{\alpha \in \mathcal{I}^{(+)}} \lambda_{\alpha}^{B}\right)^{-\frac{1}{4}} \delta_{A B}^{(\ell)}
\end{aligned}
$$

where

$$
\delta_{A B}^{(\ell)}=\sum_{\phi}\left\langle\omega^{(\ell+1)}\left[\varepsilon^{B}\right], \omega^{(\ell+1)}\left[u_{\phi}^{B}\right]\right\rangle\left\langle\omega^{(\ell)}\left[u_{\phi}^{A}\right], \omega^{(\ell)}\left[\varepsilon^{A}\right]\right\rangle
$$

with the product $\langle\bullet, \bullet\rangle$ of Eq. 3.15$)\left(G^{\alpha \beta} \simeq \delta^{\alpha \beta}\right)$.

At this stage we make some remarks.

1. The terms other than $\delta_{A B}^{(\ell)}$ are factorized into the part depending on the local data on $\gamma_{A}$ and that on $\gamma_{B}$, which may be absorbed in the normalizations of $\Omega_{\gamma_{A}}$ and $\Omega_{\gamma_{B}}$, respectively.

2. There are no zero-eigenvalues in the Hessian operators evaluated at $\gamma_{A}$ and $\gamma_{B}$. Therefore, Eq.(4.43) becomes well defined using the $\zeta$ function regularization for the positive eigenvalues.

3. $\delta_{A B}^{(\ell)}$ is interpreted as follows. Since both $u_{\alpha}^{A}$ and $e_{\alpha}^{A}(\alpha=0,1, \ldots, \ell-1)$ are the orthonormal bases of the $\ell$-dimensional negative eigenspace $N\left(\gamma_{A}\right) \subset \Gamma\left(\gamma_{A}^{*} T N\right)\left(u_{\alpha}^{B}\right.$ and $e_{\alpha}^{B}$ are in a similar situation), they are related by the orthogonal matrix $\mathcal{O}_{\phi}^{A} \in O(\ell)\left(\mathcal{O}_{\phi}^{B} \in O(\ell+1)\right)$. It follows that

$$
\omega^{(\ell)}\left[\varepsilon^{A}\right]=\left(\operatorname{det} \mathcal{O}_{\phi}^{A}\right) \omega^{(\ell)}\left[u_{\phi}^{A}\right], \quad \omega^{(\ell+1)}\left[\varepsilon^{B}\right]=\left(\operatorname{det} \mathcal{O}_{\phi}^{B}\right) \omega^{(\ell+1)}\left[u_{\phi}^{B}\right],
$$


and hence we have the expression,

$$
\delta_{A B}^{(\ell)}=\sum_{\phi} \delta_{A B}(\phi)
$$

where $\delta_{A B}(\phi)=\left(\operatorname{det} \mathcal{O}_{\phi}^{A}\right)\left(\operatorname{det} \mathcal{O}_{\phi}^{B}\right)$, which takes the values \pm 1 comparing the two orientations of $N\left(\gamma_{A}\right)$ and those of $N\left(\gamma_{B}\right)$. Following Witten's paper, ${ }^{3}$ we now define a complex $(X, \delta)$ :

$$
\begin{aligned}
X & =\left\{X^{\ell} \mid \ell=0,1, \ldots\right\}, \\
\delta & =\left\{\delta^{(\ell)}: X^{\ell} \longrightarrow X^{\ell+1} \mid \ell=0,1, \ldots\right\} .
\end{aligned}
$$

Here, $X^{\ell}$ is in Eq. (3.36) and $\delta^{(\ell)}$ the linear mapping with matrix elements $\delta_{A B}^{(\ell)}$. Thus $\delta^{(\ell)}$ is nilpotent, i.e., $\delta^{(\ell+1)} \circ \delta^{(\ell)}=0$; we can form the cohomology associated with $(X, \delta)$.

\section{EXAMPLE}

Having formulated the complex $(X, \delta)$ on the mapping space $\Omega_{p, q}(N)$ in general form, let us apply to a simple example.

We consider now $N$ to be the 2-dimensional sphere with the standard metric, $S^{2}=\left\{\left(\phi^{1}, \phi^{2}, \phi^{3}\right) \in \mathbb{R}^{3} \mid\left(\phi^{1}\right)^{2}+\left(\phi^{2}\right)^{2}+\left(\phi^{3}\right)^{2}=1\right\}$. First, remember the basic facts concerning Morse theory over $\Omega_{p, q}\left(S^{2}\right) .{ }^{19}$

(M-1) Using the local coordinates $\left(\phi^{1}, \phi^{2}\right)$ defined by the correspondence $\left(\phi^{1}, \phi^{2}, \phi^{3}\right) \longrightarrow\left(\phi^{1}, \phi^{2}\right)$, we express the metric on $S^{2}$ as

$$
g_{i j}=\delta_{i j}+\frac{\phi^{i} \phi^{j}}{1-\left(\phi^{1}\right)^{2}-\left(\phi^{2}\right)^{2}} .
$$

Then a Morse function over $\Omega_{p, q}\left(S^{2}\right)$ is defined by

$$
E[\phi]=\frac{1}{2} \int_{0}^{1} d x g_{i j} \frac{\partial \phi^{i}}{\partial x} \frac{\partial \phi^{j}}{\partial x}, \quad \phi \in \Omega_{p, q}\left(S^{2}\right),
$$

where $p=(0,0)$ and $q=(\sin \kappa, 0)(0<\kappa<\pi)$.

(M-2) The geodesics in $\Omega_{p, q}\left(S^{2}\right)$ are the paths which go round the big circle $m$ times $(m=0,1, \ldots)$ :

$$
\begin{aligned}
& \gamma_{m}^{(+)}(x)=(\sin (\kappa+2 \pi m) x, 0), \\
& \gamma_{m}^{(-)}(x)=(-\sin (\check{\kappa}+2 \pi m) x, 0) \quad(\check{\kappa}=2 \pi-\kappa)
\end{aligned}
$$

with $\gamma_{m}^{( \pm)}(0)=p$ and $\gamma_{m}^{( \pm)}(1)=q$. 
(M-3) The Morse indices of $\gamma_{m}^{(+)}$and $\gamma_{m}^{(-)}$are $2 m$ and $2 m+1$, respectively. In fact we have the following eigenfunctions of $J_{\gamma_{m}^{( \pm)}}:^{20}$

$$
e_{n}(x)=\sin n \pi x(0,1) \quad(n=1,2, \ldots)
$$

with the corresponding eigenvalues,

$$
\begin{array}{ll}
\lambda_{n}^{(+)}=(n \pi)^{2}-(\kappa+2 m \pi)^{2} & \text { for } \gamma_{m}^{(+)}, \\
\lambda_{n}^{(-)}=(n \pi)^{2}-(\check{\kappa}+2 m \pi)^{2} & \text { for } \gamma_{m}^{(-)} .
\end{array}
$$

Note that the negative eigenvalues are $\lambda_{n}^{(+)}(n=1,2, \ldots, 2 m)$ and $\lambda_{n}^{(-)}$ $(n=1,2, \ldots, 2 m+1)$.

Following the construction Eq. 3.36), we have the approximate physical Hilbert space,

$$
\mathcal{H}_{\text {phys }} \simeq \bigoplus_{\ell=0}^{\infty} X^{\ell}, \quad \operatorname{dim} X^{\ell}=1 \quad \text { for all } \ell
$$

We now determine the coboundary operator $\delta^{(\ell)}: X^{\ell} \longrightarrow X^{\ell+1}$. First, consider the 0 -th coboundary $\delta^{(0)}$. The relevant instantons are the solutions of Eq.(2.19) $\left(\Gamma_{j k}^{i}=\phi^{i} g_{j k}\right)$,

$$
\frac{\partial \phi^{i}}{\partial t}=-\frac{\partial^{2} \phi^{i}}{\partial x^{2}}-\phi^{i} g_{j k} \frac{\partial \phi^{j}}{\partial x} \frac{\partial \phi^{k}}{\partial x}
$$

satisfying the conditions $\phi(-\infty, x)=\gamma_{0}^{(+)}(x)$ and $\phi(\infty, x)=\gamma_{0}^{(-)}(x)$. Then there exist two solutions $\phi_{A}(t, x)(A=R, L$, $)$, which are opposite in sign of the second component (see Fig.1):

$$
\phi_{R}(t, x)=\left(\phi^{1}(t, x), \phi^{2}(t, x)\right), \quad \phi_{L}(t, x)=\left(\phi^{1}(t, x),-\phi^{2}(t, x)\right),
$$

which have the asymptotic behavior,

$$
\phi_{A}(t, x) \sim \begin{cases}\exp _{\gamma_{0}^{(+)}(x)}\left(c_{A}^{(+)} e^{\lambda_{1}^{(+)} t} e_{1}(x)\right) & \text { for } t \rightarrow-\infty \\ \exp _{\gamma_{0}^{(-)}(x)}\left(c_{A}^{(-)} e^{\lambda_{1}^{(-)} t} e_{1}(x)\right) & \text { for } t \rightarrow \infty\end{cases}
$$

where $c_{A}^{( \pm)}$are constants such that $c_{R}^{( \pm)}=-c_{L}^{( \pm)}$. It is easy to see that these instantons induce the opposite orientations on the 1-dimensional negative eigenspace $N\left(\gamma_{0}^{(-)}\right)$and therefore we have $\delta^{(0)}=0$. 
Next, we shall examine the coboundary $\delta^{(2 m)}: X^{2 m} \longrightarrow X^{2 m+1}(m \geq$ 1). Then the instantons of interest are the solutions of Eq.(5.9), which asymptotically satisfy the conditions $\phi(-\infty, x)=\gamma_{m}^{(+)}(x)$ and $\phi(\infty, x)=$ $\gamma_{m}^{(-)}(x)$.

These solutions are constructed as follows: First slice the interval of $x$, $I=[0,1]$, into small pieces,

$$
I_{i}=\left[\frac{i-1}{2 m+1}, \frac{i}{2 m+1}\right], \quad I=\bigcup_{i=1}^{2 m+1} I_{i} .
$$

If we put

$$
a_{i}=\gamma_{m}^{(+)}\left(\frac{i}{2 m+1}\right)=\gamma_{m}^{(-)}\left(\frac{i}{2 m+1}\right) \quad\left(a_{0}=p, a_{2 m+1}=q\right),
$$

then both of the restricted geodesics $\left.\gamma_{m}^{(+)}\right|_{I_{i}}$ and $\left.\gamma_{m}^{(-)}\right|_{I_{i}}$ express the paths which go from $a_{i-1}$ to $a_{i}$ and further can be regarded as the geodesics with the Morse indices 0 and 1, respectively. So there exist two solutions of Eq. (5.9),$\phi_{A}^{I_{i}}(A=R, L)$, connecting the geodesics $\left.\gamma_{m}^{( \pm)}\right|_{I_{i}}$.

Using these solutions as the building blocks we obtain two instantons $\phi_{A}(t, x)(A=R, L)$ (see Figs.2 and 3):

$$
\phi_{R}(t, x)= \begin{cases}\phi_{R}^{I_{1}}(t, x) & x \in I_{1}, \\ \phi_{L}^{I_{2}}(t, x) & x \in I_{2}, \\ \phi_{R}^{I_{3}}(t, x) & x \in I_{3}, \\ \vdots & \vdots \\ \phi_{R}^{I_{2 m+1}}(t, x) & x \in I_{2 m+1},\end{cases}
$$

and $\phi_{L}(t, x)$ is given by exchanging " $\mathrm{R}$ " for " $\mathrm{L}$ " in the r.h.s.. Then they have the asymptotic behavior,

$$
\phi_{A}(t, x) \sim \begin{cases}\exp _{\gamma_{m}^{(+)}(x)}\left(c_{A}^{(+)} e^{\lambda_{2 m+1}^{(+)} t} e_{2 m+1}(x)\right) & \text { for } t \rightarrow-\infty, \\ \exp _{\gamma_{m}^{(-)}(x)}\left(c_{A}^{(-)} e^{\lambda_{2 m+1}^{(-)} t} e_{2 m+1}(x)\right) & \text { for } t \rightarrow \infty,\end{cases}
$$

where $c_{A}^{( \pm)}$are constants such that $c_{R}^{( \pm)}=-c_{L}^{( \pm)}$.

By the symmetry between $\phi_{R}$ and $\phi_{L}$, we get $\delta^{(2 m)}=0$. Again using similar analyses, it follows that $\delta^{(2 m+1)}=0$, so all coboundary operators vanish. Therefore the result Eq.(5.8) in a perturbative approximation is the exact formula representing the physical Hilbert space. In fact we confirm that this formula coincides with the de Rham cohomology $H^{*}\left(\Omega_{p, q}\left(S^{2}\right)\right) .{ }^{19}$ 


\section{CONCLUDING REMARKS}

In this paper we have taken a specific topological field theory over $\Sigma=$ $\mathbb{R} \times[0,1]$, whose physical Hilbert space is given by the cohomology of the mapping space $\Omega_{p, q}(N)$. Further work that is worth considering along these lines is an examination of the topological field theory over $\Sigma=\mathbb{R} \times \mathrm{M}$ for a Riemannian manifold $(M, h)$, where the action is defined by the same formula as Eq.(2.1).

This requires the consideration of Morse theory adapted to the mapping space $\Omega(M, N)=\{\phi:(M, h) \longrightarrow(N, g)\} .{ }^{21,22}$ A natural choice of functional on $\Omega(M, N)$ is given by

$$
E[\phi]=\frac{1}{2} \int_{M} d v_{h} g_{i j}(\phi) h^{\alpha \beta}(x) \frac{\partial \phi^{i}}{\partial x^{\alpha}} \frac{\partial \phi^{j}}{\partial x^{\beta}} .
$$

Then the critical points of $E$ are harmonic maps. It is known that the Hessian operator has finite number of non-positive eigenvalues; one can associate the Morse index for each harmonic map. Thus the main problems are to construct "instantons" to interpolate between the harmonic maps and to determine the coboundary operator of the cohomology complex. This general area deserves further attention.

\section{ACKNOWLEDGMENTS}

S. Takahashi was partially supported by a Japan Ministry of Education, Science and Culture Grant-in-Aid for Scientific Research on Priority Areas (\#319), Project "Symbiotic Biosphere: An Ecological Interaction Network Promoting the Coexistence of Many Species".

Y. Yasui was partially supported by a Grant-in-Aid for Scientific Research of the Ministry of Education, Science and Culture No. 04302015.

\section{APPENDIX. EXPANSION FORMULAS}

In this appendix we prove the expansion formulas Eqs.(3.14) and (3.23). Suppose $\phi_{s}(s \in \mathbb{R})$ is a curve on $\Omega_{p, q}(N)$ such that

$$
\phi_{s}=\operatorname{Exp}_{\phi_{0}}(s \xi)=\exp \circ \sum_{\alpha=0}^{\infty} s \xi^{\alpha} e_{\alpha}, \quad \xi \in \Gamma\left(\phi_{0}^{*} T N\right) .
$$


Then, $\phi_{s}(x)(s \in \mathbb{R}, \mathrm{x} \in[0,1])$ is a family of curves on $N$ and a geodesic for any fixed $x$ :

$$
\nabla_{s} \frac{\partial \phi_{s}(x)}{\partial s}=0,\left.\quad \frac{\partial \phi_{s}(x)}{\partial s}\right|_{s=0}=\xi(x) .
$$

We denote by $\partial / \partial \phi_{s}^{\alpha}(\alpha=0,1, \ldots)$ a basis of $\Gamma\left(\phi_{s}^{*} T N\right)$ and set

$$
Y_{\alpha}(s, x)=s \frac{\partial}{\partial \phi_{s}^{\alpha}(x)}=\left.\frac{\partial}{\partial \lambda}\left(\exp \circ s\left(\xi+\lambda e_{\alpha}\right)(x)\right)\right|_{\lambda=0} .
$$

It is easy to show that each $Y_{\alpha}(s, x)$ for any fixed $x$ is a Jacobi field on $N$ along $\phi_{s}(x)$ :

$$
\nabla_{s} \nabla_{s} Y_{\alpha}(s, x)+R\left(Y_{\alpha}(s, x), \frac{\partial \phi_{s}(x)}{\partial s}\right) \frac{\partial \phi_{s}(x)}{\partial s}=0,
$$

with the initial conditions,

$$
Y_{\alpha}(0, x)=0, \quad \nabla_{s} Y_{\alpha}(0, x)=e_{\alpha}(x) .
$$

So it is immediate that

$$
\nabla_{s}^{2} Y_{\alpha}(0, x)=0, \quad \nabla_{s}^{3} Y_{\alpha}(0, x)=-R\left(e_{\alpha}(x), \xi(x)\right) \xi(x) .
$$

\section{Expansion of $G_{\alpha \beta}$}

We define $G_{\alpha \beta}(s)=\left\langle\partial / \partial \phi_{s}^{\alpha}, \partial / \partial \phi_{s}^{\beta}\right\rangle$ and so, from Eq.(A.3),

$$
s^{2} G_{\alpha \beta}(s)=\int_{0}^{1} d x\left(Y_{\alpha}(s, x), Y_{\beta}(s, x)\right) .
$$

The successive differentiations of Eq. (A.7) yield:

$$
\begin{aligned}
6 & \frac{d}{d s} G_{\alpha \beta}+6 s\left(\frac{d}{d s}\right)^{2} G_{\alpha \beta}+s^{2}\left(\frac{d}{d s}\right)^{3} G_{\alpha \beta} \\
= & \int_{0}^{1} d x\left\{\left(\nabla_{s}^{3} Y_{\alpha}, Y_{\beta}\right)+3\left(\nabla_{s}^{2} Y_{\alpha}, \nabla_{s} Y_{\beta}\right)\right. \\
& \left.+3\left(\nabla_{s} Y_{\alpha}, \nabla_{s}^{2} Y_{\beta}\right)+\left(Y_{\alpha}, \nabla_{s}^{3} Y_{\beta}\right)\right\},
\end{aligned}
$$

and

$$
\begin{aligned}
& 12\left(\frac{d}{d s}\right)^{2} G_{\alpha \beta}+8 s\left(\frac{d}{d s}\right)^{3} G_{\alpha \beta}+s^{2}\left(\frac{d}{d s}\right)^{4} G_{\alpha \beta} \\
& =\int_{0}^{1} d x\left\{\left(\nabla_{s}^{4} Y_{\alpha}, Y_{\beta}\right)+4\left(\nabla_{s}^{3} Y_{\alpha}, \nabla_{s} Y_{\beta}\right)+6\left(\nabla_{s}^{2} Y^{\alpha}, \nabla_{s}^{2} Y_{\beta}\right)\right. \\
& \left.\quad+4\left(\nabla_{s} Y_{\alpha}, \nabla_{s}^{3} Y_{\beta}\right)+\left(Y_{\alpha}, \nabla_{s}^{4} Y_{\beta}\right)\right\} .
\end{aligned}
$$


It follows from these formulas, together with Eqs. A.5) and (A.6), that the expansion of $G_{\alpha \beta}(s)$ is given by

$$
G_{\alpha \beta}(s)=\delta_{\alpha \beta}-\frac{s^{2}}{3} \int_{0}^{1} d x\left(R\left(e_{\alpha}, \xi\right) \xi, e_{\beta}\right)+\mathcal{O}\left(s^{3}\right) .
$$

Setting $s=1$, we obtain Eq.(3.14).

\section{Expansion of $\operatorname{grad} E[\phi]_{\alpha}$}

Using Eqs.(3.22) and (A.3), we have

$$
s\left(\operatorname{grad} E\left[\phi_{s}\right]_{\alpha}\right)=\int_{0}^{1} d x\left(-\nabla_{x} \frac{\partial \phi_{s}(x)}{\partial x}, Y_{\alpha}(s, x)\right) .
$$

The successive differentiations of Eq. A.11 yield:

$$
\begin{aligned}
& \operatorname{grad} E\left[\phi_{s}\right]_{\alpha}+s \frac{d}{d s} \operatorname{grad} E\left[\phi_{s}\right]_{\alpha} \\
& =\int_{0}^{1} d x\left\{\left(-\nabla_{x} \nabla_{x} \frac{\partial \phi_{s}}{\partial s}, Y_{\alpha}\right)+\left(R\left(\frac{\partial \phi_{s}}{\partial x}, \frac{\partial \phi_{s}}{\partial s}\right) \frac{\partial \phi_{s}}{\partial x}, Y_{\alpha}\right)\right. \\
& \left.\quad+\left(-\nabla_{x} \frac{\partial \phi_{s}}{\partial x}, \nabla_{s} Y_{\alpha}\right)\right\}
\end{aligned}
$$

and

$$
\begin{aligned}
& 2 \frac{d}{d s} \operatorname{grad} E\left[\phi_{s}\right]_{\alpha}+s\left(\frac{d}{d s}\right)^{2} \operatorname{grad} E\left[\phi_{s}\right]_{\alpha} \\
& =\int_{0}^{1} d x\left\{\left(-\nabla_{s} \nabla_{x} \nabla_{x} \frac{\partial \phi_{s}}{\partial s}, Y_{\alpha}\right)+2\left(-\nabla_{x} \nabla_{x} \frac{\partial \phi_{s}}{\partial s}, \nabla_{s} Y_{\alpha}\right)\right. \\
& \quad+\left(\nabla_{s}\left(R\left(\frac{\partial \phi_{s}}{\partial x}, \frac{\partial \phi_{s}}{\partial s}\right) \frac{\partial \phi_{s}}{\partial x}\right), Y_{\alpha}\right)+2\left(R\left(\frac{\partial \phi_{s}}{\partial x}, \frac{\partial \phi_{s}}{\partial s}\right) \frac{\partial \phi_{s}}{\partial x}, \nabla_{s} Y_{\alpha}\right) \\
& \left.\quad+\left(-\nabla_{x} \frac{\partial \phi_{s}}{\partial x}, \nabla_{s} \nabla_{s} Y_{\alpha}\right)\right\} .
\end{aligned}
$$

Thus the expansion of $\operatorname{grad} E\left[\phi_{s}\right]$ is given by

$$
\begin{aligned}
\operatorname{grad} E\left[\phi_{s}\right]_{\alpha}= & \int_{0}^{1} d x\left\{\left(-\nabla_{x} \frac{\partial \phi_{0}}{\partial x}, e_{\alpha}\right)-s\left(\nabla_{x} \nabla_{x} \xi+R\left(\xi, \frac{\partial \phi_{0}}{\partial x}\right) \frac{\partial \phi_{0}}{\partial x}, e_{\alpha}\right)\right\} \\
& +\mathcal{O}\left(s^{2}\right) .
\end{aligned}
$$

Setting $s=1$, we obtain Eq.(3.23). 


\section{REFERENCES AND FOOTNOTES}

1. E. Witten, Commun. Math. Phys. 117, 353 (1988); 118, 411 (1988).

2. For a review, see D. Birmingham, M. Blau, M. Rakowski, and G. Thompson, Phys. Rep. 209, 129 (1991).

3. E. Witten, J. Diff. Geom. 17, 661 (1982).

4. P. Salomonson and J. W. Van Holten, Nucl. Phys. B 196, 509 (1982).

5. F. Cooper and B. Freedman, Ann. Phys. 146, 262 (1983).

6. H. Bohr, E. Katznelson, and K. S. Narain, Nucl. Phys. B 238, 407 (1984).

7. J. M. Leinaas and K. Olaussen, Nucl. Phys. B 239,209 (1984).

8. T. Hirokane, M. Miyajima, and Y. Yasui, J. Math. Phys. 34, 2789 (1993).

9. B. Helffer and J. Sjöstrand, Commun. P.D.E. 10, 245 (1985).

10. S. Gallot, D. Hulin, and J. Lafontaine, Riemannian Geometry (Springer, New York, 1987).

11. The components of $R$ are defined by $R\left(\frac{\partial}{\partial \phi^{\imath}}, \frac{\partial}{\partial \phi^{j}}\right) \frac{\partial}{\partial \phi^{k}}=R_{k i j}^{\ell} \frac{\partial}{\partial \phi^{\ell}}$.

12. D. Birmingham, M. Rakowski, and G. Thompson, Nucl. Phys. B 315, 577 (1989).

13. I. A. Batalin and G. A. Vilkovisky, Phys. Rev. D 28,2567 (1983);

Nucl. Phys. B 234, 106 (1984); J. Math. Phys. 26, 172 (1985).

14. S. K. Ottarsson, J. Geom. Phys. 2, 49 (1985).

15. Y. Chen and M. Struwe, Math. Z. 201, 83 (1989).

16. I. M. Gelfand and A. M. Yaglom, J. Math. Phys. 1, 48 (1960).

17. H. Kleinert, Path Integrals (World Scientific, Singapore, 1990).

18. We have used the normalization such that $\left\langle\Omega_{\gamma_{I}}\left|e^{-i \widehat{H}(2 T)}\right| \Omega_{\gamma_{I}}\right\rangle=1$ for $I=A, B$. 
19. R. Bott, Amer. Math. Soc. 7, 331 (1982).

20. There exist other eigenfunctions of $J_{\gamma_{m}^{( \pm)}}$with the positive eigenvalues: $e_{n}(x)=\sin n \pi x\left(\frac{d}{d x} \gamma_{m}^{( \pm)}(x)\right)$ with $\lambda_{n}^{( \pm)}=n^{2} \pi^{2}(n=1,2, \ldots)$.

21. J. Eells, Harmonic Maps (World Scientific, Singapore, 1992).

22. K. C. Chang, Infinite Dimensional Morse Theory and Multiple Solution Problems (Birkhäuser, Boston, 1993). 


\section{FIGURE CAPTIONS}

1. Numerical calculation of two instantons $\phi_{A}(A=R, L)$ going from $\gamma_{0}^{(+)}$to $\gamma_{0}^{(-)}$. The parameter $\kappa$ is taken to be $\pi / 2: p=(0,0,1)$ and $q=(1,0,0)$ on $S^{2}$. The dotted lines of the instantons run the back side on $S^{2}$.

2. Numerical calculation of the instanton $\phi_{R}$ connecting $\gamma_{2}^{(+)}$with $\gamma_{2}^{(-)}$. The time flow is $\gamma_{2}^{(+)} \rightarrow(a) \rightarrow(b) \rightarrow \cdots \rightarrow(e) \rightarrow \gamma_{2}^{(-)}$.

3. Instanton configuration. The upper (lower) semicircles denote the instantons $\phi_{R}\left(\phi_{L}\right)$ connecting the geodesics, which are represented by "•". The numbers $(0,1, \ldots)$ denote the Morse indices associated with the geodesics. 
This figure "fig1-1.png" is available in "png" format from: http://arxiv.org/ps/hep-th/9312100v1 
This figure "fig1-2.png" is available in "png" format from: http://arxiv.org/ps/hep-th/9312100v1 
This figure "fig1-3.png" is available in "png" format from: http://arxiv.org/ps/hep-th/9312100v1 